\title{
Assessment of Islet Specificity of Dihydrotetrabenazine Radiotracer Binding in Rat Pancreas and Human Pancreas
}

Veronica Fagerholm ${ }^{1}$, Kirsi K. Mikkola ${ }^{1}$, Tamiko Ishizu ${ }^{1}$, Eveliina Arponen ${ }^{1}$, Saila Kauhanen ${ }^{1,2}$, Kjell Någren ${ }^{1}$, Olof Solin ${ }^{1}$, Pirjo Nuutila ${ }^{1}$, and Merja Haaparanta ${ }^{1}$

${ }^{I}$ Turku PET Centre, University of Turku, Turku, Finland; and ${ }^{2}$ Department of Surgery, Turku University Hospital, Turku, Finland

Vesicular monoamine transporter 2 (VMAT2) is a putative molecular target for the quantitative imaging of pancreatic $\beta$-cell mass by PET. The VMAT2 PET tracer ${ }^{11} \mathrm{C}$-dihydrotetrabenazine $\left({ }^{11} \mathrm{C}\right.$-DTBZ) exhibits high pancreatic uptake that is reduced in type 1 diabetes. The aim of this study was to assess the islet and VMAT2 specificity of DTBZ binding in the pancreas. Methods: The biodistribution of ${ }^{11} \mathrm{C}-\mathrm{DTBZ}$ in rats was determined 10 and 60 min after injection. The localization of DTBZ radioactivity in rat and human pancreatic tissue sections was investigated by autoradiography. Saturation and competition binding assays were performed with ${ }^{3} \mathrm{H}-\mathrm{DTBZ}$ and sections of rat pancreatic and control tissues. The binding of ${ }^{11} \mathrm{C}$-DTBZ in pancreatic sections from rats with streptozotocin-induced diabetes was compared with that in control rats. Results: The values for the pancreatic uptake of ${ }^{11} \mathrm{C}-\mathrm{DTBZ}$ (percentage injected dose per gram of tissue) were 3.0 at $10 \mathrm{~min}$ and 2.7 at $60 \mathrm{~min}$. At $10 \mathrm{~min}$, pancreatic radioactivity was heterogeneously distributed, with higher levels toward the head of the pancreas (head-to-tail ratio, 1.7). No such gradient was observed in pancreatic sections incubated with ${ }^{11} \mathrm{C}-\mathrm{DTBZ}$ and ${ }^{3} \mathrm{H}$-DTBZ in vitro. In rats, ${ }^{11} \mathrm{C}-\mathrm{DTBZ}$ and ${ }^{3} \mathrm{H}-\mathrm{DTBZ}$ binding in pancreatic islets did not exceed binding in the exocrine pancreas. Saturable ${ }^{3} \mathrm{H}-\mathrm{DTBZ}$ binding was observed in the rat brain striatum (dissociation constant $\left[K_{d}\right], 1.3 \mathrm{nM}$ ) and the bovine adrenal medulla $\left(\mathrm{K}_{\mathrm{d}}, 3.3 \mathrm{nM}\right)$, whereas in the rat pancreas, ${ }^{3} \mathrm{H}$-DTBZ binding was nonsaturable. Competition binding with ${ }^{3} \mathrm{H}$-DTBZ and VMAT2 antagonists also indicated that DTBZ binding in the rat pancreas was nonspecific and did not represent binding to VMAT2. Nonspecific pancreatic ${ }^{11} \mathrm{C}$-DTBZ binding was lower in rats with streptozotocininduced diabetes than in control rats. In sections of human pancreas, a subset of pancreatic islets were weakly but VMAT2-specifically labeled with ${ }^{3} \mathrm{H}$-DTBZ. Conclusion: The results showed that the pancreatic uptake of ${ }^{11} \mathrm{C}$-DTBZ is mainly due to nonspecific binding in the exocrine pancreas and suggested that the reduction in pancreatic ${ }^{11} \mathrm{C}-\mathrm{DTBZ}$ binding observed in type 1 diabetes is not specific for the loss of $\beta$-cell mass.

Received Dec. 31, 2009; revision accepted Mar. 24, 2010.

For correspondence or reprints contact: Veronica Fagerholm, Turku PET Centre, MediCity Research Laboratories, Tykistökatu 6A, FIN-20520 Turku, Finland.

E-mail: veronica.fagerholm@utu.fi

COPYRIGHT $\odot 2010$ by the Society of Nuclear Medicine, Inc.
Key Words: autoradiography; $\beta$-cell mass; DTBZ; pancreas; PET

J Nucl Med 2010; 51:1439-1446

DOI: 10.2967/jnumed.109.074492

$\mathbf{P}$ and adult hyperinsulinism $(1,2)$ and pancreatic cancer, including pancreatic islet cell tumors $(3,4)$, are already in clinical use. In addition, efforts have been made to develop PET tracers specific for pancreatic $\beta$-cells, which would provide a noninvasive method for estimating $\beta$-cell mass. The loss of pancreatic $\beta$-cells is a feature of both type 1 and advanced type 2 diabetes. At present, estimations of pancreatic $\beta$-cell integrity rely on measurements of stimulated insulin secretion capacity.

Two distinct vesicular monoamine transporters, vesicular monoamine transporter 1 and vesicular monoamine transporter 2 (VMAT2), transport biogenic amines into intracellular storage vesicles (5-7). Neurons and pancreatic $\beta$-cells accumulate monoamines by using VMAT2 (8-10), the pharmacology of which has been extensively characterized with bovine adrenal medulla chromaffin granules (11). The VMAT2 antagonist ${ }^{11} \mathrm{C}$-dihydrotetrabenazine $\left({ }^{11} \mathrm{C}\right.$ DTBZ) is an established tracer for PET imaging of the dopaminergic system of the brain (12). High uptake of ${ }^{11} \mathrm{C}$-DTBZ radioactivity is evident in the pancreas of nondiabetic rodents. PET studies in rats showed that the uptake of ${ }^{11} \mathrm{C}$-DTBZ radioactivity in the pancreas declined with the onset of autoimmune and streptozotocin-induced diabetes $(13,14)$. Moreover, the pancreatic uptake of ${ }^{11} \mathrm{C}$-DTBZ radioactivity was lower in a group of patients with long-standing type 1 diabetes than in a group of healthy volunteers, as determined by PET (15). Therefore, ${ }^{11} \mathrm{C}$-DTBZ has been considered to be a candidate molecule for the noninvasive quantitative imaging of pancreatic $\beta$-cell mass in humans $(16,17)$. However, the localization of ${ }^{11} \mathrm{C}$-DTBZ radioactivity specifically to the pancreatic islets has not been verified.

This investigation was performed to determine the islet and VMAT2 specificity of DTBZ binding in rat pancreas 
and human pancreas. After injection of ${ }^{11} \mathrm{C}-\mathrm{DTBZ}$ into rats, pancreatic tissue sections were prepared, and the accumulation of radioactivity in pancreatic islets and the exocrine pancreas was assessed. The effect of type 1 diabetes on ${ }^{11} \mathrm{C}$ DTBZ binding in the pancreas was studied in vitro with pancreatic sections from rats with streptozotocin-induced diabetes. Saturation and competition binding assays were performed with ${ }^{3} \mathrm{H}$-DTBZ and sections of rat pancreatic tissues and VMAT2-rich control tissues to determine the VMAT2 specificity of pancreatic DTBZ binding. ${ }^{3} \mathrm{H}-\mathrm{DTBZ}$ autoradiography was used to assess binding to pancreatic islets in humans.

\section{MATERIALS AND METHODS}

\section{Chemicals}

$(+)-\alpha-{ }^{11} \mathrm{C}-\mathrm{DTBZ}\left({ }^{11} \mathrm{C}\right.$-DTBZ) was synthesized by ${ }^{11} \mathrm{C}$ methylation of (+)-9-O-desmethyl- $\alpha$-dihydrotetrabenazine by a previously described method (18), with minor modifications. The radiochemical purity of the final product exceeded $98 \% . \alpha-2-{ }^{3} \mathrm{H}-$ DTBZ with a specific radioactivity of $0.74 \mathrm{MBq} / \mathrm{nmol}$ was purchased from American Radiolabeled Chemicals. Chlorpromazine, haloperidol, ketanserin, lobeline, reserpine, streptozotocin, and tetrabenazine were obtained from Sigma-Aldrich Co.

\section{Experimental Animals and Tissues from \\ Human Patients}

Sprague-Dawley male rats (200-350 g), bred and housed under standard conditions at the Central Animal Laboratory, University of Turku, Turku, Finland, were used. Bovine adrenal gland was obtained from a local slaughterhouse (Paimion Teurastamo Paimio, Finland). The animal experiments were approved by the Animal Care and Use Committee of the University of Turku and by the State Provincial Office of Southern Finland. Human pancreatic tissue was obtained from 4 patients who underwent pancreatic resection at Turku University Hospital (Turku, Finland). The patients, 3 women and 1 man, were $59 \pm 12$ (mean \pm SD) y old, were not diabetic, and had pancreatic cystadenoma or pancreatic cystadenocarcinoma. Approval for the use of human pancreatic tissue was obtained from the Ethics Committee of the Hospital District of Southwest Finland. All patients gave written informed consent before participating in the study.

\section{Biodistribution and Intrapancreatic Localization of ${ }^{11} \mathrm{C}-\mathrm{DTBZ}$ Radioactivity}

Rats $(n=14)$ weighing $269 \pm 45 \mathrm{~g}$ were briefly anesthetized with $\mathrm{CO}_{2}: \mathrm{O}_{2}$ (50:50) gas and injected via a tail vein with ${ }^{11} \mathrm{C}-\mathrm{DTBZ}$ at $170 \pm 105 \mathrm{MBq} / \mathrm{kg}$. The specific radioactivity at the time of injection was $155 \pm 93 \mathrm{MBq} / \mathrm{nmol}$, and the injected molecular mass was $2.1 \pm 2.2 \mathrm{nmol} / \mathrm{kg}$. At $10 \mathrm{~min}(n=7)$ or $60 \mathrm{~min}(n=7)$ after injection, the rats were anesthetized with $\mathrm{CO}_{2}$ gas, and blood was collected by cardiac puncture. Plasma, tissues, and organs were weighed, and their radioactivities were measured. The biodistribution of the radioactivity, corrected for radionuclide decay, was reported as the percentage injected dose per gram of tissue.

The pancreata and brains were frozen by immersion in isopentane chilled with dry ice and then were sectioned at 20 $\mu \mathrm{m}$ on a cryostat. The sections were dried under a fan and apposed to imaging plates (Fuji BAS-TR2025; Fuji Photo Film Co.) for approximately $1 \mathrm{~h}$. Digital images were obtained by scanning of the imaging plates with a BAS-5000 scanner (Fuji Photo Film Co.) at a $25-\mu \mathrm{m}$ resolution or an FLA-5100 scanner (Fuji Photo Film Co.) at a $10-\mu \mathrm{m}$ resolution. Visual inspection of the pancreatic images suggested that at $10 \mathrm{~min}$ after the injection of ${ }^{11} \mathrm{C}$-DTBZ, the radioactivity levels were higher toward the head than toward the tail of the pancreas. The most distal $5 \mathrm{~mm}$ at each end of the pancreatic sections was outlined and analyzed with image analysis software (Aida 4; Raytest), and the results were reported as photostimulated luminescence per square millimeter. Three sections per rat ( $n=6,10$-min time point) were evaluated. The pancreatic head-to-tail binding ratio was determined for each rat, and the results were statistically analyzed by comparing the head-to-tail ratios to hypothetical value 1 .

Pancreatic sections that had been processed for autoradiography were stained with hematoxylin and eosin, and the distributions of ${ }^{11} \mathrm{C}$-DTBZ radioactivity and pancreatic islets were compared by visual inspection ( $n=3,10$-min time point, $2-4$ sections per rat). The results were documented by taking a high-magnification composite photograph of a stained section with a $10 \times$ objective on a Zeiss Axiovert $200 \mathrm{M}$ microscope (Carl Zeiss Inc.) equipped with a digital camera, a motorized stage, and software for the generation of mosaic images. Pancreatic islets were identified and manually outlined on the micrographs, and the outlines were combined with the autoradiographic images by use of Photoshop CS3 (Adobe Systems Inc.).

\section{Metabolite Analysis}

A rat was injected with ${ }^{11} \mathrm{C}-\mathrm{DTBZ}$ at $361 \mathrm{MBq} / \mathrm{kg}(3.8 \mathrm{nmol} / \mathrm{kg})$; the specific radioactivity at the time of injection was $222 \mathrm{MBq} / \mathrm{nmol}$. The presence of radioactive metabolites in plasma, urine, liver, and pancreas 10 min after injection was assessed by thin-layer chromatography. The head, tail, and middle portions of the pancreas were analyzed separately. ${ }^{11} \mathrm{C}$-DTBZ added to rat plasma was used as a standard to allow identification of the parent compound. Plasma and urine proteins were precipitated with 1.25 times the sample volume of acetonitrile and removed by centrifugation. The tissue samples were homogenized in acetonitrile: $\mathrm{H}_{2} \mathrm{O}$ (30:70), and the radioactive material was purified in 2 centrifugation steps. Supernatants $(10 \mu \mathrm{L})$ were applied to an HPTLC Silica Gel 60 plate (105547; Merck). Chloroform:methanol (85:15), $2 \times 10 \mathrm{~mL}$, was used as the mobile phase. The vapor space was presaturated in a twin developing chamber (Camag Twin Through Chamber; Camag) for $30 \mathrm{~min}$. The plate was developed $4 \mathrm{~cm}$, dried under a fan, and apposed to an imaging plate for approximately $1 \mathrm{~h}$ as described earlier.

\section{Induction of Diabetes and In Vitro Binding of ${ }^{11} \mathrm{C}-\mathrm{DTBZ}$ in Pancreatic Sections from Diabetic and Control Rats}

On day 1, 2 groups of rats, weight-matched on day 0 , were fasted for $4 \mathrm{~h}$. Blood glucose from a drop of blood from a tail vein was measured with an ACCU-CHEK Aviva glucose meter (Roche Diagnostics) and plasma-calibrated test strips. The 2 groups of rats were anesthetized with isoflurane, and either streptozotocin $(65 \mathrm{mg} / \mathrm{kg})$ dissolved in $0.1 \mathrm{M}$ citrate buffer $(\mathrm{pH} \mathrm{4.6)}(n=8)$ or citrate buffer alone $(n=6)$ was intravenously administered. Blood glucose levels and body weights were monitored regularly and compared with baseline values obtained on day 1 . The control rats remained euglycemic $(7.0 \pm 0.7 \mathrm{mM}$ on day 8$)$, and their body weights increased from $332 \pm 19 \mathrm{~g}$ on day 0 to $346 \pm 16 \mathrm{~g}$ on day 8. Two of the rats in the streptozotocin group displayed only moderate elevations in blood glucose levels, and their body 
weights increased. These rats were excluded from the study. The rest of the rats in the streptozotocin group $(n=6)$ exhibited blood glucose levels exceeding $22 \mathrm{mM}$ from day 3 onward, and their body weights decreased from $329 \pm 21 \mathrm{~g}$ on day 1 to $283 \pm$ $22 \mathrm{~g}$ on day 7 . On day 7 (streptozotocin group) or day 8 (control group), the rats were decapitated under isoflurane anesthesia, and their pancreata were dissected and frozen. Pancreatic sections, $20 \mu \mathrm{m}$ thick, were cut on a cryostat and stored frozen until used.

Two pancreatic sections from each animal were incubated with $2.8 \mathrm{nM}{ }^{11} \mathrm{C}$-DTBZ (at a specific radioactivity of $180 \mathrm{MBq} / \mathrm{nmol}$ at the start of incubation) in $50 \mathrm{mM}$ potassium phosphate buffer $(\mathrm{pH}$ 7.4) at room temperature for $20 \mathrm{~min}$. The sections were washed in ice-cold buffer for $10 \mathrm{~min}$, rinsed in ice-cold distilled water, dried under a fan, and apposed to imaging plates (Fuji BASTR2025) for approximately $1 \mathrm{~h}$. Whole pancreatic sections were manually outlined and analyzed as described earlier. Occasional hot-spot artifacts, not corresponding to pancreatic islets (e.g., lower left-hand corners of Figs. 3B and 3C), were excluded from the regions of interest. Results from duplicate sections were averaged, and the levels of binding in diabetic and control rats were compared.

\section{Pharmacologic Characterization of ${ }^{3} \mathrm{H}-\mathrm{DTBZ}$ Binding In Vitro}

${ }^{3} \mathrm{H}-\mathrm{DTBZ}$ saturation and competition binding assays were performed by incubating duplicate sections of rat pancreas, rat brain, and bovine adrenal gland with ${ }^{3} \mathrm{H}$-DTBZ and various unlabeled compounds in $50 \mathrm{mM}$ potassium phosphate buffer for $2 \mathrm{~h}$ at room temperature. In the saturation binding experiment, sections from 1 rat and 1 bull were incubated with 11 concentrations of ${ }^{3} \mathrm{H}-\mathrm{DTBZ}$, ranging from 0.38 to $47.3 \mathrm{nM}$. Adjacent sections were incubated in the presence of an excess of a VMAT2 antagonist $(100 \mu \mathrm{M}$ ketanserin) to determine nonspecific binding. The competition binding experiment was performed with $1.8 \mathrm{nM}{ }^{3} \mathrm{H}-\mathrm{DTBZ}$ and 7 concentrations of unlabeled compounds, ranging from $0 \mathrm{nM}$ to $0.1 \mathrm{mM}$. The pancreata from 2 rats, the brain from 1 rat, and the adrenal glands from 1 cow and 1 bull were used in the competition binding experiment. Because similar results were obtained from the cow and the bull, their corresponding binding values were averaged.

After the incubations, the sections were washed for $20 \mathrm{~min}$ in ice-cold buffer, rapidly rinsed in ice-cold distilled water, and dried under a fan. A 20-min wash was found to be optimal on the basis of the ${ }^{3} \mathrm{H}$-DTBZ binding ratios for the rat brain striatum to the cerebral cortex and the bovine adrenal medulla to the adrenal cortex after different washing times (data not shown). The dry sections were apposed to imaging plates (Fuji BAS-TR2025) for 10 d. ${ }^{3} \mathrm{H}$ standards (ART-123; ARC Inc.) were coexposed with the sections to allow comparisons of the results from separate experiments. ${ }^{3} \mathrm{H}$-DTBZ binding levels in the rat pancreas, rat striatum (bilateral readings), and bovine adrenal medulla were measured. Results from duplicate sections were averaged.

Equilibrium dissociation $\left(\mathrm{K}_{\mathrm{d}}\right)$ and inhibition $\left(\mathrm{K}_{\mathrm{i}}\right)$ constants were determined by least squares nonlinear regression analysis of the binding results with GraphPad Prism 5 (GraphPad Software). The saturation binding results were analyzed with simultaneous fitting of total and nonspecific binding. $\mathrm{K}_{\mathrm{d}}$ values obtained from the saturation binding experiment were used to calculate $K_{i}$ values for the unlabeled compounds applied in the competition binding experiment; for this purpose, we used the Cheng-Prusoff equation (18): $\mathrm{K}_{\mathrm{i}}=\mathrm{IC}_{50} /(1+$ [radioligand $\left.] / \mathrm{K}_{\mathrm{d}}\right)$, where $\mathrm{IC}_{50}$ is the $50 \%$ inhibitory concentration.

\section{${ }^{3} \mathrm{H}-\mathrm{DTBZ}$ Binding in Human Pancreas and Insulin Immunohistochemistry Analysis}

Human pancreas sections $(n=4)$ were frozen immediately after surgery by immersion in isopentane chilled with dry ice. Duplicate sections, $20 \mu \mathrm{m}$ thick, were incubated with $5.4 \mathrm{nM}$ ${ }^{3} \mathrm{H}$-DTBZ for $30 \mathrm{~min}$ in the absence or presence of $100 \mu \mathrm{M}$ ketanserin. Sections of rat brain and bovine adrenal medulla were included as controls. The sections were washed for $20 \mathrm{~min}$, dried, and exposed to imaging plates for $14 \mathrm{~d}$. After autoradiographic exposure, the sections were fixed with $4 \%$ paraformaldehyde and processed for insulin immunohistochemistry analysis with a Vectastain ABC Kit (Vector Laboratories). A polyclonal guinea pig primary antibody raised against human insulin (Abcam), diluted $1: 500$, and then a biotinylated goat anti-guinea pig secondary antibody (Jackson ImmunoResearch), diluted 1:500, were used. The sections were counterstained with hematoxylin and photographed as described earlier. Pancreatic islets were identified and manually outlined on the micrographs, and the outlines were combined with the autoradiographic images by use of CorelDRAW X3 (Corel Corp.).

\section{Statistics}

Results were analyzed with Microsoft Excel 2002 (Microsoft Corp.) and GraphPad Prism 5 and reported as mean \pm SD or as mean and $95 \%$ confidence interval. Differences between groups were assessed with a 2-tailed Student $t$ test. Differences between ratios and 1 were analyzed with a 2-tailed 1-sample $t$ test. A $P$ value of less than 0.05 was considered statistically significant.

\section{RESULTS}

\section{Biodistribution and Intrapancreatic Localization of ${ }^{11} \mathrm{C}-\mathrm{DTBZ}$ Radioactivity in Rats}

The largest accumulation of ${ }^{11} \mathrm{C}$-DTBZ radioactivity was found in the pancreas, with similarly high levels at 10 and $60 \mathrm{~min}$ after injection (Table 1). In addition, the striatum exhibited high uptake of radioactivity $10 \mathrm{~min}$ after injection, but at $60 \mathrm{~min}$, radioactivity in the striatum was significantly reduced; these results suggest different kinetics for ${ }^{11} \mathrm{C}$-DTBZ binding in the pancreas and the VMAT2-rich striatum. The amounts of nonmetabolized tracer, as determined by thin-layer chromatography $10 \mathrm{~min}$ after the injection of ${ }^{11} \mathrm{C}$-DTBZ, were $94 \%$ in plasma, $85 \%$ in urine, $83 \%$ in liver, $98 \%$ in the head of the pancreas, $99 \%$ in the middle of the pancreas, and $98 \%$ in the tail of the pancreas. Two to 3 radioactive metabolites were separated; their $R_{f}$ values were lower than the $R_{f}$ value of ${ }^{11} \mathrm{C}$-DTBZ, indicating that the metabolites were more polar than the parent compound (data not shown). The possible formation of radioactive volatile metabolites was not assessed.

A representative example of autoradiographic and histologic images of a pancreatic section, with pancreatic islets outlined, is shown in Figure 1. At $10 \mathrm{~min}$ after the injection of ${ }^{11} \mathrm{C}$-DTBZ, radioactivity was heterogeneously distributed; levels toward the head of the pancreas were $1.7 \pm$ 0.6 -fold (i.e., $70 \%$ ) higher than those toward the tail $(P=$ $0.046, n=6)$. At 60 min after injection, this gradient was 


\section{TABLE 1. ${ }^{11} \mathrm{C}$-DTBZ Biodistribution in Rats 10 and 60 Minutes After Injection}

\begin{tabular}{|c|c|c|c|}
\hline Organ or tissue & $10 \min (n)$ & $60 \min (n)$ & $P^{\star}$ \\
\hline Pancreas & $3.03 \pm 0.62(7)$ & $2.74 \pm 0.59(7)$ & 0.38 \\
\hline Striatum & $2.92 \pm 0.97(6)$ & $1.17 \pm 0.43(5)$ & 0.005 \\
\hline Liver & $2.08 \pm 0.44(3)$ & $1.87 \pm 0.41(7)$ & 0.26 \\
\hline Thymus & $1.56 \pm 0.37(3)$ & $0.39 \pm 0.08(7)$ & $<0.001$ \\
\hline Adrenal gland & $1.44 \pm 0.09(2)$ & $0.59 \pm 0.11(7)$ & $<0.001$ \\
\hline Stomach wall & $1.38 \pm 0.37(2)$ & $0.47 \pm 0.12(6)$ & $<0.001$ \\
\hline Salivary gland & $1.28 \pm 0.12(3)$ & $0.93 \pm 0.11(7)$ & 0.002 \\
\hline Kidney & $1.16 \pm 0.27(3)$ & $0.59 \pm 0.08(7)$ & $<0.001$ \\
\hline Whole brain & $1.05 \pm 0.48(7)$ & $0.35 \pm 0.06(7)$ & 0.002 \\
\hline Thyroid gland & $1.02 \pm 0.31(3)$ & $0.40 \pm 0.01$ & 0.009 \\
\hline Cerebral cortex & $0.89 \pm 0.40(7)$ & $0.25 \pm 0.04$ & 0.006 \\
\hline Stomach $^{\dagger}$ & $0.85 \pm 0.12(2)$ & $0.25 \pm 0.04(6)$ & 0.006 \\
\hline Lung & $0.79 \pm 0.11$ & $2.74 \pm 0.59(7)$ & 0.005 \\
\hline Small intestine ${ }^{\dagger}$ & $0.77 \pm 0.16$ & $1.29 \pm 0.21(7)$ & 0.001 \\
\hline Large intestine $^{\dagger}$ & $0.77 \pm 0.16(3)$ & $0.15 \pm 0.04(7)$ & $<0.001$ \\
\hline Heart & $0.73 \pm 0.10(3)$ & $0.28 \pm 0.04(7)$ & $<0.001$ \\
\hline Cerebellar cortex & $0.73 \pm 0.29(7)$ & $0.24 \pm 0.07(5)$ & 0.12 \\
\hline Spleen & $0.69 \pm 0.09(3)$ & $0.48 \pm 0.05(7)$ & 0.99 \\
\hline Bone marrow & $0.65 \pm 0.13(3)$ & $0.52 \pm 0.09(6)$ & 0.78 \\
\hline Eye & $0.46 \pm 0.16$ & $0.15 \pm 0.05(7)$ & 0.15 \\
\hline Testis & $0.46 \pm 0.03(3)$ & $0.38 \pm 0.08(7)$ & $<0.001$ \\
\hline Mesenteric fat & $0.23 \pm 0.13(3)$ & $0.22 \pm 0.03(7)$ & $<0.001$ \\
\hline Bone & $0.22 \pm 0.06(3)$ & $0.09 \pm 0.01$ & 0.74 \\
\hline Skeletal muscle & $0.22 \pm 0.01(3)$ & $0.13 \pm 0.02(7)$ & 0.22 \\
\hline Skin & $0.21 \pm 0.03$ & $0.20 \pm 0.03(7)$ & 0.002 \\
\hline Blood cells & $0.19 \pm 0.02(7)$ & $0.10 \pm 0.01(7)$ & $<0.001$ \\
\hline Whole blood & $0.17 \pm 0.02(7)$ & $0.10 \pm 0.01(7)$ & $<0.001$ \\
\hline Plasma & $0.15 \pm 0.02(7)$ & $0.10 \pm 0.01(7)$ & $<0.001$ \\
\hline
\end{tabular}

no longer seen. At either time point, ${ }^{11} \mathrm{C}-\mathrm{DTBZ}$ radioactivity did not accumulate specifically in pancreatic islets. In the rat brain, the expected ${ }^{11} \mathrm{C}$-DTBZ binding pattern, corresponding to the known distribution of VMAT2, was observed (data not shown).

In Vitro Binding of ${ }^{11} \mathrm{C}-\mathrm{DTBZ}$ in Pancreatic Sections from Diabetic and Control Rats

${ }^{11} \mathrm{C}$-DTBZ binding in the pancreas was lower in diabetic rats than in control rats (Fig. 2A), supporting previous findings obtained by in vivo PET. The decrease in ${ }^{11} \mathrm{C}$-DTBZ binding was observed throughout the pancreatic tissue, indicating that it was not directly associated with a decrease in $\beta$-cell mass (Figs. 2B and 2C).

\section{Pharmacologic Characterization of ${ }^{3} \mathrm{H}-\mathrm{DTBZ}$ Binding In Vitro}

${ }^{3} \mathrm{H}-\mathrm{DTBZ}$ binding in sections of the rat pancreas was homogeneous, and pancreatic islets could not be visualized. In the rat pancreas, ${ }^{3} \mathrm{H}-\mathrm{DTBZ}$ binding increased in a concentration-dependent, nonsaturable manner (Fig. 3). In contrast, saturable ${ }^{3} \mathrm{H}-\mathrm{DTBZ}$ binding was observed in the rat striatum $\left(\mathrm{K}_{\mathrm{d}}, 1.3 \mathrm{nM}\right.$; 95\% confidence interval, $\left.1.0-1.5 \mathrm{nM}\right)$ and bovine adrenal medulla $\left(\mathrm{K}_{\mathrm{d}}, 3.3 \mathrm{nM} ; 95 \%\right.$ confidence interval, 2.1-4.6 nM). In the rat striatum and bovine adrenal medulla, VMAT2 antagonists competed for ${ }^{3} \mathrm{H}-\mathrm{DTBZ}$ binding with the following rank order of potency: tetrabenazine $>$ ketanserin $>$ reserpine $>$ haloperidol $>$ lobeline $\approx$ chlorpromazine; this profile corresponded to the pharmacologic profile of VMAT2 $(11,20,21)$. All competitors exhibited somewhat higher affinities for the rat striatum than for the bovine adrenal medulla (Table 2). A non-VMAT2 competition binding profile was observed in the rat pancreas, with the following rank order of potency: lobeline $\approx$ ketanserin $>$ haloperidol $\approx$ chlorpromazine $>$ tetrabenazine $\approx$ reserpine. The confidence intervals were wide, however, and reliable $50 \%$ inhibitory concentrations could not be determined. This situation was partly attributable to the poor displacement of pancreatic ${ }^{3} \mathrm{H}$-DTBZ binding by the unlabeled compounds and to the lower levels of ${ }^{3} \mathrm{H}$-DTBZ binding in the pancreas than in the striatum and adrenal medulla at the concentration of ${ }^{3} \mathrm{H}$-DTBZ used $(1.8 \mathrm{nM})$. Together, the in vitro binding results suggest that ${ }^{3} \mathrm{H}$-DTBZ binding in the rat pancreas, in contrast to binding in the rat striatum and bovine adrenal medulla, is nonspecific.

\section{${ }^{3} \mathrm{H}-\mathrm{DTBZ}$ Binding in Human Pancreas}

Insulin-immunoreactive pancreatic islets were observed in samples from all patients. ${ }^{3} \mathrm{H}$-DTBZ binding in most 

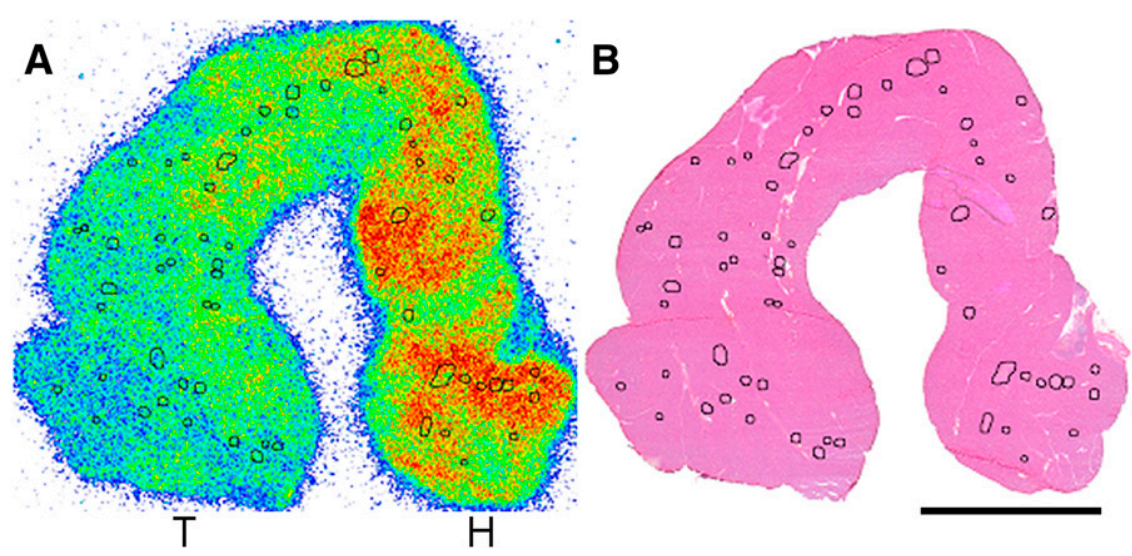

FIGURE 1. Intrapancreatic distribution of radioactivity $10 \mathrm{~min}$ after injection of ${ }^{11} \mathrm{C}-\mathrm{DTBZ}$ at $1.0 \mathrm{nmol} / \mathrm{kg}$. Same pancreatic section used for autoradiography $(A)$ was stained with hematoxylin and eosin (B). ${ }^{11} \mathrm{C}-\mathrm{DTBZ}$ radioactivity did not accumulate in pancreatic islets (outlined in A and B). Radioactivity gradient with higher levels toward head of pancreas, as seen in A, was consistently observed at $10 \mathrm{~min}$ but not at $60 \mathrm{~min}$ after injection. Scale bar $=5$ $\mathrm{mm}$. $\mathrm{H}=$ head of pancreas; $\mathrm{T}=$ tail of pancreas. islets did not exceed binding in the exocrine pancreas. Some intermediate to large islets were, however, discernible against the exocrine background labeling (Fig. 4). Ketanserin at $100 \mu \mathrm{M}$ completely inhibited islet-specific ${ }^{3} \mathrm{H}-\mathrm{DTBZ}$ binding, suggesting that it represented binding to VMAT2. At the ${ }^{3} \mathrm{H}-\mathrm{DTBZ}$ concentration used $(5.4 \mathrm{nM})$, the labeling intensity in human islets was less than 2-fold the intensity in the surrounding exocrine tissue. For comparison, the ratio for the binding of the rat striatum to the rat pancreas was 3.3, that for the binding of the rat striatum to the rat cerebral cortex was 6.7, and that for the binding of the bovine adrenal medulla to the bovine adrenal cortex was 6.8. The levels of nonspecific binding were similar in rat exocrine pancreas and human exocrine pancreas.

\section{DISCUSSION}

The results of the present study demonstrated that the binding of ${ }^{11} \mathrm{C}-\mathrm{DTBZ}$ and ${ }^{3} \mathrm{H}$-DTBZ in islets of the rat pancreas did not exceed the binding in exocrine tissue. Pancreatic binding increased rapidly with increasing concentrations of ${ }^{3} \mathrm{H}-\mathrm{DTBZ}$ but did not represent binding to VMAT2. Nonspecific pancreatic ${ }^{11} \mathrm{C}-\mathrm{DTBZ}$ binding was lower in rats with streptozotocin-induced diabetes than in control rats. In the human pancreas, only weak ${ }^{3} \mathrm{H}-\mathrm{DTBZ}$ labeling of a small number of islets was detected.

Although $\beta$-cells, which reside in pancreatic islets scattered throughout the exocrine tissue, constitute only a small fraction of the total pancreatic volume, the highest levels of radioactivity after the injection of ${ }^{11} \mathrm{C}$-DTBZ into rats were found in the pancreas. Similar levels of pancreatic uptake of
${ }^{3} \mathrm{H}-\mathrm{DTBZ}$ and ${ }^{11} \mathrm{C}-\mathrm{DTBZ}$ as well as DTBZ derivatives were reported previously $(13,14,22,23)$. In those studies, the islet and VMAT2 specificity of the accumulated radioactivity was not assessed directly. If pancreatic ${ }^{11} \mathrm{C}-\mathrm{DTBZ}$ uptake represented primarily binding to $\beta$-cells, then strong labeling of pancreatic islets would be expected. However, we did not observe islet-specific accumulation of radioactivity in the rat pancreas either after intravenous injection of ${ }^{11} \mathrm{C}$-DTBZ or under in vitro conditions with ${ }^{11} \mathrm{C}$-DTBZ or ${ }^{3} \mathrm{H}$-DTBZ; under such conditions, the concentrations of radiolabeled DTBZ could be strictly controlled, and in vivo metabolism of the tracer was not a concern. ${ }^{11} \mathrm{C}-\mathrm{DTBZ}$ and ${ }^{3} \mathrm{H}-\mathrm{DTBZ}$ displayed the expected binding patterns in the rat brain and bovine adrenal gland, demonstrating the integrity of the tracers under the binding conditions used. Transiently higher radioactivity levels toward the head than toward the tail of the rat pancreas were consistently observed $10 \mathrm{~min}$ after the injection of ${ }^{11} \mathrm{C}$-DTBZ. Because the heterogeneous distribution of radioactivity did not correspond to the distribution of pancreatic islets, the possibility of regional differences in either blood flow or blood perfusion or binding to sympathetic nerves remains. The fact that a binding gradient was not observed in pancreatic sections incubated with ${ }^{11} \mathrm{C}$-DTBZ or ${ }^{3} \mathrm{H}$-DTBZ in vitro argues against the latter alternative.

Pharmacologic comparisons of ${ }^{3} \mathrm{H}-\mathrm{DTBZ}$ binding in sections of the rat pancreas, rat striatum, and bovine adrenal medulla confirmed the notion that DTBZ binding in the rat pancreas did not represent binding to VMAT2. At concentrations of less than $10 \mathrm{nM}$, total ${ }^{3} \mathrm{H}$-DTBZ binding was
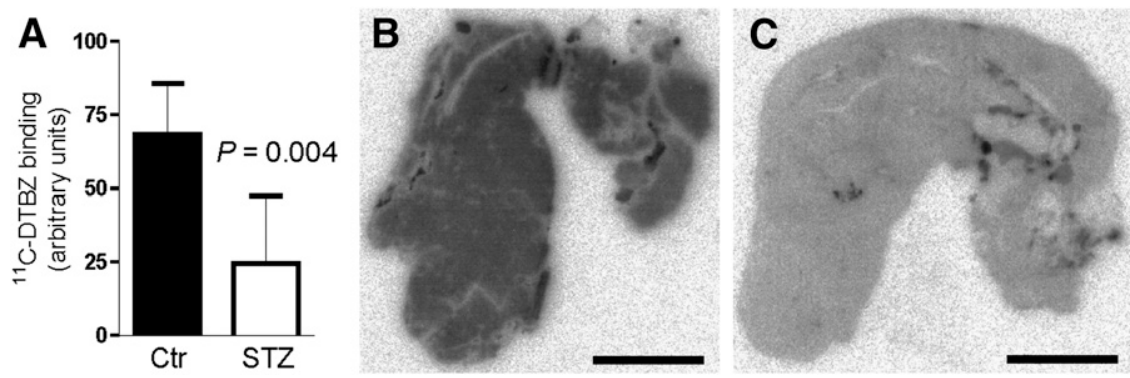

FIGURE 2. (A) Pancreatic ${ }^{11} \mathrm{C}-\mathrm{DTBZ}$ $(2.8 \mathrm{nM})$ binding was lower in sections from diabetic (STZ) rats $(n=6)$ than in sections from control (Ctr) rats $(n=6)$ (Student $t$ test). Sections from STZ and Ctr rats were processed in parallel in same experiment. (B and C) Representative sections from control rat $(\mathrm{B})$ and diabetic rat $(\mathrm{C})$. Scale bar $=5 \mathrm{~mm}$. 

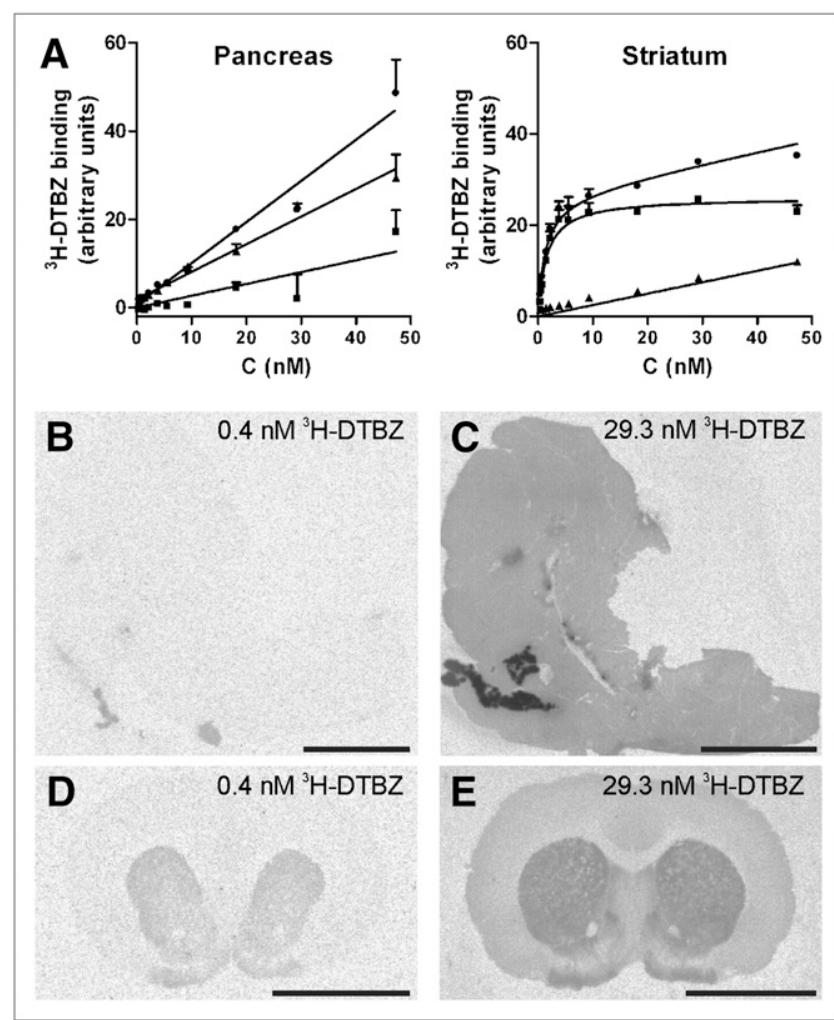

FIGURE 3. (A) ${ }^{3} \mathrm{H}$-DTBZ binding curves for sections of pancreas and striatum from same rat, processed in parallel. Nonspecific binding was defined as binding in presence of $100 \mu \mathrm{M}$ ketanserin. Specific binding ( $(\boldsymbol{\nabla})$ was difference between total binding $(\bullet)$ and nonspecific binding $(\boldsymbol{\Delta})$. Pancreatic ${ }^{3} \mathrm{H}-\mathrm{DTBZ}$ binding was nonsaturable within investigated concentration range. In striatum, ${ }^{3} \mathrm{H}-\mathrm{DTBZ}$ binding was saturable, with $\mathrm{K}_{\mathrm{d}}$ of $1.3 \mathrm{nM}$. (B-E) Relative increases in pancreatic ( $B$ and $C$ ) and striatal ( $D$ and $E$ ) binding were observed with increasing concentrations of ${ }^{3} \mathrm{H}$-DTBZ. Scale bar $=5 \mathrm{~mm}$.

considerably lower in the rat pancreas than in the rat striatum, whereas at $50 \mathrm{nM}$, the opposite was true. The amount of ${ }^{11} \mathrm{C}$-DTBZ administered to living rats was $2.1 \pm 2.2$ $\mathrm{nmol} / \mathrm{kg}$. It is conceivable that during the bolus intravenous injection, the amount of ${ }^{11} \mathrm{C}$-DTBZ that reached the pancreas was large enough to explain the abundant (non-
VMAT2) pancreatic uptake of ${ }^{11} \mathrm{C}$-DTBZ observed in the biodistribution study. Nonspecific DTBZ binding in the exocrine pancreas was not readily reversible, as evident from the slow clearance of ${ }^{11} \mathrm{C}-\mathrm{DTBZ}$ in vivo and the poor displacement of ${ }^{3} \mathrm{H}-\mathrm{DTBZ}$ by VMAT2 antagonists in vitro.

Previous assumptions that the pancreatic uptake of radiolabeled DTBZ and its derivatives represented binding to VMAT2 were based on indirect evidence. In the human pancreas, VMAT2 is found in endocrine cells and sympathetic nerve fibers, as demonstrated by in situ hybridization and immunohistochemistry analysis (9). In the endocrine human pancreas, VMAT2 is mainly localized to $\beta$-cells, although a subset of cells immunoreactive for pancreatic polypeptide was also reported to express VMAT2 (24). Mei et al. (25) reported the loss of VMAT2-immunoreactive sympathetic fibers in pancreatic islets at the onset of autoimmune but not streptozotocin-induced type 1 diabetes in rats. In that report, VMAT2 immunostaining was not evident in islets. In contrast, Souza et al. (13) and Simpson et al. (14) described decreases in islet VMAT2 and insulin immunoreactivity in association with reduced pancreatic VMAT2 and insulin messenger RNA expression in both rats with streptozotocin-induced diabetes and rats with autoimmune type 1 diabetes. These reductions were correlated with reduced pancreatic uptake of ${ }^{11} \mathrm{C}-\mathrm{DTBZ}$, as determined by PET. We found similar reductions in ${ }^{11} \mathrm{C}$-DTBZ binding in pancreatic sections from rats with streptozotocin-induced diabetes, but because the reduction in binding occurred uniformly throughout the pancreatic tissue, it could not be attributed to a decrease in $\beta$-cell mass. Supporting evidence was provided by a recent report in which sleeve gastrectomy and duodenal-jejunal bypass surgery in Goto-Kakizaki type 2 diabetic rats increased pancreatic ${ }^{11} \mathrm{C}$-DTBZ uptake and circulating insulin levels in association with a decrease, rather than an increase, in $\beta$-cell mass (26). The mechanism for the reduction of non-VMAT2 DTBZ binding in the exocrine pancreas in rats with streptozotocin-induced or autoimmune type 1 diabetes is still unknown. In rats with autoimmune diabetes, the reduction in pancreatic ${ }^{11} \mathrm{C}-\mathrm{DTBZ}$ uptake preceded the onset of diabetes (13), suggesting that hyperglycemia may not be necessary for the downregulation of DTBZ binding sites.

\section{TABLE 2. Pharmacology of ${ }^{3} \mathrm{H}-\mathrm{DTBZ}$ Binding}

\begin{tabular}{|c|c|c|c|c|}
\hline \multirow[b]{2}{*}{ Competitor } & \multicolumn{2}{|c|}{ Rat striatum $(n=1)$} & \multicolumn{2}{|c|}{ Bovine adrenal medulla $(n=2)$} \\
\hline & $\mathrm{K}_{\mathrm{i}}(\mathrm{nM})$ & $95 \% \mathrm{Cl}$ & $\mathrm{K}_{\mathrm{i}}(\mathrm{nM})$ & $95 \% \mathrm{Cl}$ \\
\hline Tetrabenazine & 8.8 & $5.7-13.6$ & 27.0 & $17.3-42.0$ \\
\hline Ketanserin & 19.2 & $12.5-29.4$ & 313 & $50.8-1,929$ \\
\hline Reserpine & 189 & $115-310$ & 936 & $467-1,873$ \\
\hline Haloperidol & 1,123 & $440-2,871$ & 5,258 & $1,862-14,850$ \\
\hline Lobeline & 4,809 & $2,750-8,407$ & $>10,000$ & ND \\
\hline Chlorpromazine & 9,194 & $3,175-26,620$ & $>10,000$ & ND \\
\hline
\end{tabular}



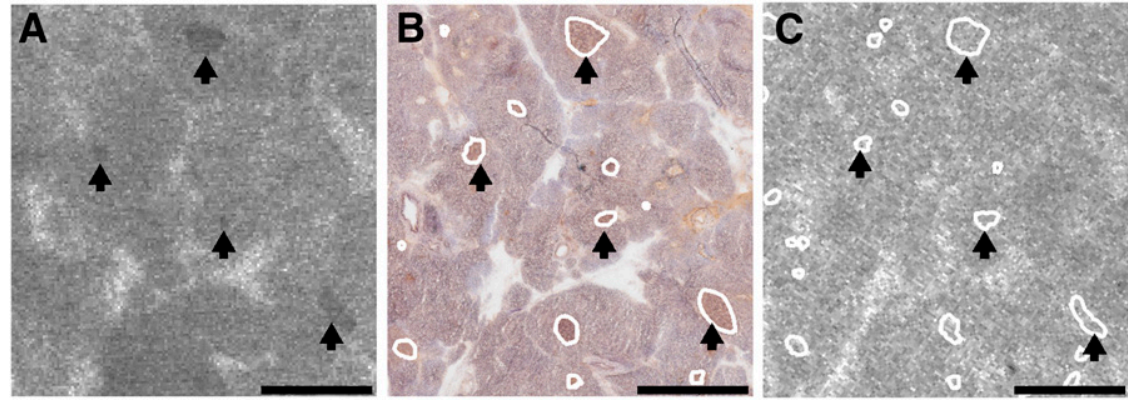

FIGURE 4. ${ }^{3} \mathrm{H}-\mathrm{DTBZ}$ binding in sections of human pancreas. (A) Arrows indicate subset of pancreatic islets that were labeled with ${ }^{3} \mathrm{H}$-DTBZ $(5.4 \mathrm{nM})$. (B) Islets were identified and outlined after insulin immunohistochemistry analysis was performed on same section. (C) Adjacent section incubated with ${ }^{3} \mathrm{H}$-DTBZ $(5.4 \mathrm{nM})$ and $100 \mu \mathrm{M}$ ketanserin. Islets labeled with ${ }^{3} \mathrm{H}-\mathrm{DTBZ}$ in $A$ were also visible in $C$. Absence of islet-specific binding in $\mathrm{C}$ indicated that labeling of islets with ${ }^{3} \mathrm{H}$-DTBZ in A was VMAT2-specific. Pancreatic islets in $\mathrm{C}$ were identified by insulin immunohistochemistry analysis (not shown) of same section. Scale bar $=1 \mathrm{~mm}$.

Although some islets were weakly labeled with ${ }^{3} \mathrm{H}-$ DTBZ in sections of the human pancreas, ${ }^{3} \mathrm{H}$-DTBZ binding in the great majority of islets did not exceed binding in the exocrine pancreas. Similar levels of nonspecific ${ }^{3} \mathrm{H}-$ DTBZ binding were observed in rat exocrine pancreas and human exocrine pancreas. This finding is in agreement with the residual pancreatic uptake of ${ }^{11} \mathrm{C}$-DTBZ detected by PET in patients with long-standing type 1 diabetes (15), expected to be devoid of $\beta$-cells. Together, these results imply that the pancreatic uptake of ${ }^{11} \mathrm{C}$-DTBZ in humans also represents mainly nonspecific binding. Extrapolating from our results for rats, we suggest that this nonspecific binding may be reduced in type 1 diabetes, resulting in the misinterpretation of PET results. Recently, radiolabeled derivatives of DTBZ with a higher affinity for VMAT2, including ${ }^{18} \mathrm{~F}-9$-fluoropropyl-(+)-DTBZ and ${ }^{18} \mathrm{~F}-(+)-2-$ oxiranyl-3-isobutyl-9-(3-fluoropropoxy)-10-methoxy-2,3,4, 6,7,11b-hexahydro-1H-pyrido[2,1-a]isoquinoline, were developed as alternative VMAT2 PET tracers. A higher affinity for VMAT2 could improve the labeling of pancreatic islets. However, because ${ }^{11} \mathrm{C}-\mathrm{DTBZ}$ and its derivatives displayed similarly high levels of pancreatic uptake when injected into rats $(22,23)$, the derivatives are likely to exhibit the same type of nonspecific pancreatic binding as the parent compound.

\section{CONCLUSION}

The results of the present study suggested that ${ }^{11} \mathrm{C}$ DTBZ cannot be used for the quantitative imaging of pancreatic $\beta$-cell mass. Further studies are needed to determine whether the observed changes in nonspecific pancreatic ${ }^{11} \mathrm{C}$-DTBZ binding in association with type 1 diabetes and, possibly, with other pathologic or physiologic conditions will provide some biologically or clinically relevant information. In addition, the possibility that VMAT2 tracers other than ${ }^{11} \mathrm{C}$-DTBZ will be better suited for the imaging of pancreatic $\beta$-cells remains.

\section{ACKNOWLEDGMENTS}

The technical assistance of Tarja Marttila, Marko Vehmanen, and Elisa Riuttala is acknowledged. The research leading to these results has received funding from the European Community's Seventh Framework Program (FP7/ 2007-2013) under grant agreement 222980 ("Use of Innovative Strategies for $\beta$-Cell Imaging in Diabetes Mellitus") and from the Diabetes Research Foundation, Tampere, Finland. This study was conducted within the Finnish Centre of Excellence in Molecular Imaging in Cardiovascular and Metabolic Research, which is financially supported by the Academy of Finland, University of Turku, Turku University Hospital, and Åbo Akademi University.

\section{REFERENCES}

1. Otonkoski T, Näntö-Salonen K, Seppänen M, et al. Noninvasive diagnosis of focal hyperinsulinism of infancy with $\left[{ }^{18} \mathrm{~F}\right]$-DOPA positron emission tomography. Diabetes. 2006;55:13-18.

2. Kauhanen S, Seppänen M, Minn H, et al. Fluorine-18-L-dihydroxyphenylalanine $\left({ }^{18} \mathrm{~F}-\mathrm{DOPA}\right)$ positron emission tomography as a tool to localize an insulinoma or betacell hyperplasia in adult patients. J Clin Endocrinol Metab. 2007;92:1237-1244.

3. Virgolini I, Traub-Weidinger T, Decristoforo C. Nuclear medicine in the detection and management of pancreatic islet-cell tumours. Best Pract Res Clin Endocrinol Metab. 2005;19:213-227.

4. Koopmans KP, Neels ON, Kema IP, et al. Molecular imaging in neuroendocrine tumors: molecular uptake mechanisms and clinical results. Crit Rev Oncol Hematol. 2009;71:199-213.

5. Liu Y, Peter D, Roghani A, et al. A cDNA that suppresses $\mathrm{MPP}^{+}$toxicity encodes a vesicular amine transporter. Cell. 1992;70:539-551.

6. Erickson JD, Eiden LE, Hoffman BJ. Expression cloning of a reserpine-sensitive vesicular monoamine transporter. Proc Natl Acad Sci USA. 1992;89:10993-10997.

7. Peter D, Jimenez J, Liu Y, Kim J, Edwards RH. The chromaffin granule and synaptic vesicle amine transporters differ in substrate recognition and sensitivity to inhibitors. J Biol Chem. 1994;269:7231-7237.

8. Erickson JD, Schäfer MK, Bonner TI, Eiden LE, Weihe E. Distinct pharmacological properties and distribution in neurons and endocrine cells of two isoforms of the human vesicular monoamine transporter. Proc Natl Acad Sci USA. 1996;93:5166-5171.

9. Anlauf M, Eissele R, Schäfer MK, et al. Expression of the two isoforms of the vesicular monoamine transporter (VMAT1 and VMAT2) in the endocrine pancreas and pancreatic endocrine tumors. J Histochem Cytochem. 2003; 51:1027-1040.

10. Maffei A, Liu Z, Witkowski P, et al. Identification of tissue-restricted transcripts in human islets. Endocrinology. 2004;145:4513-4521.

11. Scherman D, Jaudon P, Henry JP. Characterization of the monoamine carrier of chromaffin granule membrane by binding of $\left[2-{ }^{3} \mathrm{H}\right]$ dihydrotetrabenazine. Proc Natl Acad Sci USA. 1983;80:584-588.

12. Efange SM. In vivo imaging of the vesicular acetylcholine transporter and the vesicular monoamine transporter. FASEB J. 2000;14:2401-2413.

13. Souza F, Simpson N, Raffo A, et al. Longitudinal noninvasive PET-based beta cell mass estimates in a spontaneous diabetes rat model. J Clin Invest. 2006;116:1506-1513.

14. Simpson NR, Souza F, Witkowski P, et al. Visualizing pancreatic beta-cell mass with [ ${ }^{11}$ C]DTBZ. Nucl Med Biol. 2006;33:855-864. 
15. Goland R, Freeby M, Parsey R, et al. ${ }^{11} \mathrm{C}$-dihydrotetrabenazine PET of the pancreas in subjects with long-standing type 1 diabetes and in healthy controls. J Nucl Med. 2009;50:382-389.

16. Freeby M, Goland R, Ichise M, Maffei A, Leibel R, Harris P. VMAT2 quantitation by PET as a biomarker for beta-cell mass in health and disease. Diabetes Obes Metab. 2008;10(suppl. 4):98-108.

17. Harris PE, Ferrara C, Barba P, Polito T, Freeby M, Maffei A. VMAT2 gene expression and function as it applies to imaging beta-cell mass. $\mathrm{J} \mathrm{Mol} \mathrm{Med}$. 2008;86:5-16.

18. Cheng Y, Prusoff WH. Relationship between the inhibition constant (K1) and the concentration of inhibitor which causes 50 per cent inhibition (I50) of an enzymatic reaction. Biochem Pharmacol. 1973;22:3099-3108.

19. Chan GL, Holden JE, Stoessl AJ, et al. Reproducibility studies with ${ }^{11} \mathrm{C}-\mathrm{DTBZ}$, a monoamine vesicular transporter inhibitor in healthy human subjects. $J$ Nucl Med. 1999;40:283-289.

20. Darchen F, Scherman D, Laduron PM, Henry JP. Ketanserin binds to the monoamine transporter of chromaffin granules and of synaptic vesicles. Mol Pharmacol. 1988;33:672-677.
21. Teng L, Crooks PA, Dwoskin LP. Lobeline displaces $\left[{ }^{3} \mathrm{H}\right]$ dihydrotetrabenazine binding and releases $\left[{ }^{3} \mathrm{H}\right]$ dopamine from rat striatal synaptic vesicles: comparison with D-amphetamine. J Neurochem. 1998;71:258-265.

22. Kung HF, Lieberman BP, Zhuang ZP, et al. In vivo imaging of vesicular monoamine transporter 2 in pancreas using an ${ }^{18} \mathrm{~F}$ epoxide derivative of tetrabenazine. Nucl Med Biol. 2008;35:825-837.

23. Kung MP, Hou C, Lieberman BP, et al. In vivo imaging of beta-cell mass in rats using ${ }^{18} \mathrm{~F}-\mathrm{FP}-(+)-\mathrm{DTBZ}$ : a potential PET ligand for studying diabetes mellitus. J Nucl Med. 2008;49:1171-1176.

24. Saisho Y, Harris PE, Butler AE, et al. Relationship between pancreatic vesicular monoamine transporter 2 (VMAT2) and insulin expression in human pancreas. J Mol Histol. 2008;39:543-551.

25. Mei Q, Mundinger TO, Lernmark A, Taborsky GJ Jr. Early, selective, and marked loss of sympathetic nerves from the islets of BioBreeder diabetic rats. Diabetes. 2002;51:2997-3002.

26. Inabnet $\mathrm{WB}$, Milone $\mathrm{L}$, Harris $\mathrm{P}$, et al. The utility of $\left[{ }^{11} \mathrm{C}\right]$ dihydrotetrabenazine positron emission tomography scanning in assessing beta-cell performance after sleeve gastrectomy and duodenal-jejunal bypass. Surgery. 2010;147:303-309. 